\title{
Status of Vaccine Coverage In Venezuelan Children, A Country With A Complex Humanitarian Crisis
}

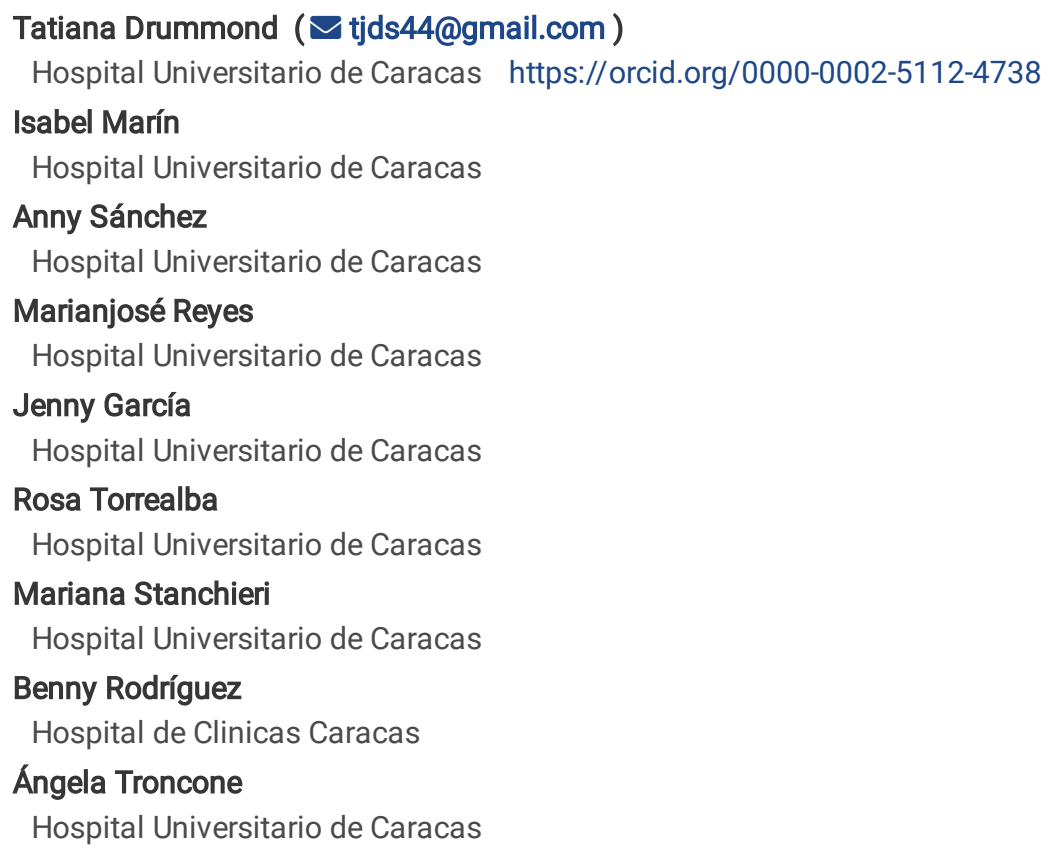




\section{Abstract}

In Venezuela, PAHO has reported an increase in vaccine-preventable diseases since 2016. The goal of this work was to assess vaccination coverage in children hospitalized in the Department of Pediatrics at the Hospital Universitario de Caracas (HUC).

Methods: A descriptive cross-sectional study included 0 to 12 years old children hospitalized in HUC admitted between January 2015 and December 2019, and verified immunization scheme. The patient data were compared with the schedule of the Ministry of Health of Venezuela and analyzed by comparing immunization coverage by year of patient hospitalization and patient age.

Results: A total of 2903 patients were surveyed, corresponding to $53.2 \%$ male, $37.4 \%$ infants. A coverage level above $95 \%$ was found only for BCG. Comparing vaccination coverage with the vaccination schedule vs year of patient hospitalization, it was observed a mean decrease in vaccine coverage of $21.5 \%$ in 2019 relative to $2015(p=0.0000)$. Vaccination rates in children under one year old were lower than in children older than 6 years for all vaccines $(p=0.0000)$

Conclusions: There is a decline in vaccination coverage in 2019 in relation to previous years, being the most affected children less than one year old

\section{Background}

Vaccination has been one of the measures with the greatest impact on public health in the last half century, not only for its effectiveness but also for its profitability, greatly contributing to two-thirds reduction in child mortality between 1990 and $2015(1,2)$. However, there is a gap between the potential use of this practice and its actual contribution to child survival (2). According to the World Health Organization (WHO), by 2017 the global rate of vaccination coverage was $86 \%$ They calculated that global vaccination coverage could be improved to prevent 1.5 million deaths, and estimated that there are still 19.5 million infants worldwide who do not receive basic vaccinations, with children in developing countries being the most affected (3).

In developing countries, low vaccination rates are multifactorial (1). Factors that interfere with vaccination coverage can be grouped in four areas: i) immunization system, i.e. structure for vaccine distribution (e.g. limited funding for routine immunization services, barriers to access to primary care); ii) parental knowledge and attitudes about vaccination programs; iii) communication and information $(2,4-8)$; and iv) family characteristics, including poverty, rurality, extremes of maternal age, multiple childbirths, low maternal education, family size and lack of knowledge about vaccine-preventable diseases $(2,4,5,8)$.

The Expanded Program on Immunization (EPI) was established in 1974 as an initiative of the WHO / Pan American Health Organization (PAHO) to improve the availability of vaccines worldwide, with the aim of ensuring that all children have access to and receive basic immunizations $(1,7)$. Systematic vaccination programs have allowed the eradication of smallpox, the interruption of transmission of polio and control of tetanus, diphtheria, rubella and invasive bacterial infection by Haemophilus influenzae type $B$ and Streptococcus pneumoniae (7).

In the EPI, monitoring and evaluation are key tools that contribute to the effective and efficient implementation of actions, since they serve to periodically verify that the work is being developed according to the plan, to identify causes of failure to meet targets and to take timely and appropriate steps to correct the deviations. (9)

Vaccination coverage measures the proportion of children who have received the vaccines according to the scheme for their age and are therefore protected (9). The minimum percentage needed to achieve herd immunity varies by disease from $75-95 \%(2,7)$.

Coverage reported in 2015 inVenezuela in the Annual Report of the Ministerio del Poder Popular para la Salud (MPPS) were: oral polio 91\%, hepatitis B 91\%, pentavalent: 91\%, MMR 97\%, yellow fever $92 \%, 87 \%$ BCG, rotavirus $88 \%$, TT-TD pregnant: $38 \%$ (10).

According to the report of PAHO's Executive Committee (included on the agenda of the Session 162), "PAHO's Response to Maintaining an Effective Technical Cooperation Agenda in Venezuela and Neighboring Member States", the burden of disease in Venezuela has become more complex and extended and diverse, affecting in particular fragile and vulnerable population. Emerging diseases have not been properly addressed, allowing the re-emergence of infectious diseases in epidemics spread of infectious diseases preventable by vaccination which had been controlled, revealing the inefficiency of epidemiological surveillance and the weakness of the national immunization program (11). The case of the measles epidemic, reemerging disease with indigenous cases since the first week of July 2017 is highlighted in the document, signaling the failure of mass vaccination campaigns. This results in an important setback which impacts Venezuela and the entire American Continent, which loses the recognition of being free of endemic transmission of the disease, reached since 2016 (11).

The purpose of this study is to evaluate vaccination coverage and its evolution from 2015 until 2018 in pediatric patients attending the University Hospital of Caracas. 


\section{Methods}

A descriptive cross-sectional study of vaccination coverage in pediatric patients, which were included all children hospitalized in Pediatric and Medical Pediatric Infectious Hospital Universitario de Caracas (HUC) aged between 1 day and 11 years, 11 months and 29 days, in the period January 2015-December 2019, whose immunization scheme could be assessed by their immunization card. For each patient, demographic data, immunization schedule and year of hospitalization in the HUC were recorded.

Immunization coverage was supposed when a child was correctly and fully vaccinated, following the Venezuelan national immunization schedule established by the Ministry of Popular Power for Health (MPPS) (12), which includes:

- Newborn: a single dose of BCG (Bacillus Calmette-Guérin)

- Under 1 year old: 3 doses of pentavalent vaccine (tetanus toxoid, diphtheria toxoid, Bordetella pertussis, Haemophilus influenzae typeB, hepatitis B), 2 doses of rotavirus vaccine, two doses of pneumococcal vaccine, 3 doses of polio vaccine and two doses of seasonal influenza vaccine. Since July 2017 a dose of viral bivalent vaccine (measles and rubella MR) was included, as ordered by the MPPS in order to control the epidemic of measles in Venezuela for that year

- 1 to 5 years: two doses of viral trivalent vaccine (MMR: measles, rubella and mumps), reinforcing Pneumococcal a yellow fever vaccine, pentavalent vaccine booster, polio vaccine booster, annual influenza vaccine.

- Older than 6 years: DT vaccine booster (tetanus and diphtheria toxoid), annual influenza vaccine

All patients whose immunization schedule could not be verified were excluded from the study.

For evaluation of vaccine coverage 3 groups were considered in response to vaccination cycles. First, patients under 12 months old (basic immunization); a second group involves patients between 12 months and 6 years old, when the reinforcement of different vaccines must be The third included patients older than 6 years, which should already have the basic immunization scheme and reinforcements for the different vaccines considered in the study.

Data were collected through the database of the Pediatric Department of HUC created for collection of egress data of pediatric patients, approved by the ethics committee HUC of 2015, and which was developed using the application Google Drive Forms. The data was analyzed descriptively by frequency and percentages for the qualitative data, and mean and standard deviation for quantitative data; Chi-square was evaluated for comparison the vaccine coverage according to age groups, year of patient egress and the combination of patient age groups vs year of hospitalization; a cutoff value for significance of $p<0.05$ was established. For statistical data processing the software Epiinfo 7.2 was used.

\section{Results}

From January 2015 to December 2019 a total of 3235 patients were registered, meeting the criteria for entry and egress a total of 2903 patients; 332 patients were excluded because they did not have complete data of the immunizations. 1545 (53.2\%) were male. Group under 12 months old corresponded to $37.4 \%$ ( $n=1085$ patients); the group between 12 months and 6 years old included $38.2 \%$ ( $n=1109$ ). The social class Graffar V (extreme poverty) was the most frequent $75.6 \%(n=2195)$. (Table 1). 
Table 1

Sample characterization

\begin{tabular}{|c|c|c|}
\hline Demographic characteristics & $\mathbf{n}$ & $\%$ \\
\hline \multicolumn{3}{|l|}{ Sex } \\
\hline Female & 1358 & $46,8 \%$ \\
\hline Male & 1545 & $53,2 \%$ \\
\hline \multicolumn{3}{|l|}{ Age group } \\
\hline$<6$ months & 757 & $26,1 \%$ \\
\hline 6 to 11 months 29 days & 328 & $11,3 \%$ \\
\hline 12 months to 6 years & 1109 & $38,2 \%$ \\
\hline$>6$ years & 709 & $24,4 \%$ \\
\hline \multicolumn{3}{|l|}{ Graffar } \\
\hline I & 2 & $0,1 \%$ \\
\hline II & 10 & $0,3 \%$ \\
\hline III & 125 & $4,3 \%$ \\
\hline IV & 571 & $19,7 \%$ \\
\hline V & 2195 & $75,6 \%$ \\
\hline \multicolumn{3}{|l|}{ Hospitalization year } \\
\hline 2015 & 644 & $22,2 \%$ \\
\hline 2016 & 634 & $21,8 \%$ \\
\hline 2017 & 695 & $23,9 \%$ \\
\hline 2018 & 547 & $18,8 \%$ \\
\hline 2019 & 383 & $13,2 \%$ \\
\hline TOTAL & 2903 & 100.0 \\
\hline
\end{tabular}

The vaccine with largest general coverage level was BCG (Bacille Calmette-Guérin) with $94.9 \%(n=2745)$ followed by polio $81.8 \%$ ( $n=2028$ ). The vaccines with lowest coverage were influenza 17.7\% $(n=332)$ and pneumococcal conjugate with $14.6 \%$ coverage $(n=332)($ Table 2$)$.

Table 2

Vaccination coverage as type of vaccine

\begin{tabular}{|lllllll|}
\hline Vaccine & \multicolumn{2}{l}{ Complete } & \multicolumn{2}{l|}{ Incomplete } & \multicolumn{2}{l|}{ Total } \\
\cline { 2 - 7 } & $\mathbf{n}$ & $\%$ & $\mathbf{n}$ & $\%$ & $\mathbf{n}$ & $\%$ \\
\hline BCG & 2745 & $94,9 \%$ & 148 & $5,1 \%$ & 2893 & $100,0 \%$ \\
\hline Polio & 2028 & $81,8 \%$ & 451 & $18,2 \%$ & 2479 & $100,0 \%$ \\
\hline Pentavalent & 1808 & $75,8 \%$ & 576 & $24,2 \%$ & 2384 & $100,0 \%$ \\
\hline Rotavirus & 1726 & $71,9 \%$ & 676 & $28,1 \%$ & 2402 & $100,0 \%$ \\
\hline Pneumococcus & 332 & $14,6 \%$ & 1948 & $85,4 \%$ & 2280 & $100,0 \%$ \\
\hline Influenza & 332 & $17,7 \%$ & 1545 & $82,3 \%$ & 1877 & $100,0 \%$ \\
\hline MMR & 1346 & $75,1 \%$ & 447 & $24,9 \%$ & 1793 & $100,0 \%$ \\
\hline Yellow fever & 1028 & $61,8 \%$ & 635 & $38,2 \%$ & 1663 & $100,0 \%$ \\
\hline
\end{tabular}

Assessing vaccination coverage considering the year of hospitalization of the patient, a decline in coverage is evident from 2015 to 2019 in all vaccines (between 6 and 52\%), being statistically significant in all cases $(p=0.0000)$. The largest coverage decline was observed for the case of rotavirus vaccination, which drop from87.9\% $(n=510)$ in 2015 to $35.7 \%(n=100)$ in $2019(p=0.0000)$. The least significant decline was 
observed for the BCG coverage, from 99.1\% $(n=637)$ in 2015 to $92.8 \%(n=349)$ for the year 2019. Pentavalent, MMR and pneumococcal vaccines experienced a decline of $20.3 \%, 16.1 \%$ and $16.2 \%$ from 2015 to 2019 (Table 3).

Table 3

Vaccination coverage and year hospitalization of patient

\begin{tabular}{|c|c|c|c|c|c|c|c|c|c|c|c|c|}
\hline \multirow[t]{2}{*}{ Vaccine } & \multicolumn{2}{|c|}{2015} & \multicolumn{2}{|c|}{2016} & \multicolumn{2}{|c|}{2017} & \multicolumn{2}{|c|}{2018} & \multicolumn{2}{|c|}{2019} & \multirow[t]{2}{*}{$p$} & \multirow[t]{2}{*}{ Chi2 } \\
\hline & $\mathrm{n}$ & $\%$ & $\mathrm{n}$ & $\%$ & $\mathrm{n}$ & $\%$ & $\mathbf{n}$ & $\%$ & $\mathbf{n}$ & $\%$ & & \\
\hline BCG & 637 & $99.10 \%$ & 605 & $95.70 \%$ & 656 & $94.40 \%$ & 498 & $91.00 \%$ & 349 & $92.8 \%$ & 0.0000 & 44.3916 \\
\hline Polio & 518 & $87.4 \%$ & 448 & $85.2 \%$ & 503 & $86.3 \%$ & 344 & $75.4 \%$ & 215 & $67.0 \%$ & 0.0000 & 83.9357 \\
\hline Pentavalent & 461 & $80.2 \%$ & 404 & $80.0 \%$ & 437 & $79.7 \%$ & 319 & $71.9 \%$ & 187 & $59.9 \%$ & 0.0000 & 62.1557 \\
\hline Rotavirus & 510 & $87.9 \%$ & 434 & $83.8 \%$ & 425 & $73.5 \%$ & 257 & $57.6 \%$ & 100 & $35.7 \%$ & 0.0000 & 336.8869 \\
\hline Pneumococcus & 120 & $21.5 \%$ & 93 & $19.6 \%$ & 60 & $11.5 \%$ & 43 & $10.0 \%$ & 16 & $5.4 \%$ & 0.0000 & 62.2331 \\
\hline Influenza & 138 & $28.5 \%$ & 91 & $23.6 \%$ & 68 & $16.8 \%$ & 17 & $4.8 \%$ & 18 & $7.4 \%$ & 0.0000 & 106.6313 \\
\hline MMR & 346 & $77.2 \%$ & 307 & $83.0 \%$ & 323 & $80.8 \%$ & 215 & $67.0 \%$ & 155 & $61.0 \%$ & 0.0000 & 58.3705 \\
\hline Yellow fever & 294 & $66.2 \%$ & 252 & $75.0 \%$ & 246 & $69.3 \%$ & 130 & $43.2 \%$ & 106 & $46.7 \%$ & 0.0000 & 103.0292 \\
\hline
\end{tabular}

Regarding the different age groups, the group under 12 months old had lower coverage in all vaccines than other groups, with $87.4 \%$ ( $n=939$ ), coverage for BCG compared with $99.0 \%(n=1098)$ for children 1 to 6 years of age and $99.9 \%(n=708)$ for children older than 6 years $(p=$ 0.0000). For polio vaccine coverage was $58.3 \%(n=396)$ for children under 1 year old, while for children aged 1 to 6 years and older than 6 was $88.0 \%(n=965)$ and $94.9 \%(n=667)$ respectively $(p=0.0000)$. The greatest difference between age groups was observed in the case of rotavirus vaccine: while the coverage was $39.3 \%(n=251)$ for the group under one year, it was $91.4 \%(n=634)$ for children older than 6 years $(p$ $=0.0000)$ (Table 4).

Table 4

Patient age vs immunization coverage

\begin{tabular}{|c|c|c|c|c|c|c|c|c|}
\hline \multirow[t]{2}{*}{ Vaccine } & \multicolumn{2}{|c|}{ < 1 year } & \multicolumn{2}{|c|}{$\geq 1$ to 6 years } & \multicolumn{2}{|c|}{$\geq 6$ years } & \multirow[t]{2}{*}{$\mathbf{P}$} & \multirow[t]{2}{*}{ Chi2 } \\
\hline & $\mathbf{n}$ & $\%$ & $\mathbf{n}$ & $\%$ & $\mathbf{n}$ & $\%$ & & \\
\hline BCG & 939 & $87.4 \%$ & 1098 & $99.0 \%$ & 708 & $99.9 \%$ & 0.0000 & 200.7531 \\
\hline Polio & 396 & $58.3 \%$ & 965 & $88.0 \%$ & 667 & $94.9 \%$ & 0.0000 & 360.3340 \\
\hline Pentavalent & 334 & $53.4 \%$ & 864 & $80.9 \%$ & 610 & $88.4 \%$ & 0.0000 & 182.1056 \\
\hline Rotavirus & 251 & $39.3 \%$ & 841 & $78.6 \%$ & 634 & $91.4 \%$ & 0.0000 & 488.0485 \\
\hline Pneumococcus & 61 & $10.0 \%$ & 116 & $11.6 \%$ & 155 & $23.3 \%$ & 0.0000 & 58.5141 \\
\hline Influenza & $15^{\star}$ & $6.2 \% *$ & 170 & $17.3 \%$ & 147 & $22.7 \%$ & 0.0000 & 33.2324 \\
\hline MMR & $30 *$ & $31.3 \% *$ & 729 & $71.6 \%$ & 587 & $86.5 \%$ & 0.0000 & 151.9962 \\
\hline
\end{tabular}

By correlating the age group with the year of hospitalization and vaccination coverage it can be observed that the age group under 1 year old had the largest drop in immunization coverage when comparing the years 2015 through 2019; the largest decline was observed for the rotavirus vaccine, with $69.2 \%$ coverage decrease between 2015 and $2019(p=0.0000)$. (Table 5) 
Table 5

Vaccination coverage by year of hospitalization and patient age

\begin{tabular}{|c|c|c|c|c|c|c|c|}
\hline & 2015 & 2016 & 2017 & 2018 & 2019 & $\mathbf{P}$ & Chi2 \\
\hline & $\%$ & $\%$ & $\%$ & $\%$ & $\%$ & & \\
\hline \multicolumn{8}{|l|}{ Under 1 year } \\
\hline BCG & $97.1 \%$ & $89.6 \%$ & $85.6 \%$ & $81.9 \%$ & $84.2 \%$ & 0.0001 & 24.5656 \\
\hline Polio & $74.8 \%$ & $66.9 \%$ & $56.1 \%$ & $48.0 \%$ & $43.6 \%$ & 0.0000 & 34.5134 \\
\hline Pentavalent & $73.3 \%$ & $64.4 \%$ & $47.1 \%$ & $46.81 \%$ & $30.9 \%$ & 0.0000 & 49.6378 \\
\hline Rotavirus & $76.5 \%$ & $62.9 \%$ & $30.5 \%$ & $12.1 \%$ & $7.3 \%$ & 0.0000 & 185.7668 \\
\hline Pneumococcus & $26.7 \%$ & $15.3 \%$ & $2.9 \%$ & $2.2 \%$ & $3.5 \%$ & 0.0000 & 61.7479 \\
\hline Influenza* & $23.10 \%$ & $8.3 \%$ & $4.0 \%$ & 0.0 & $0.0 \%$ & 0.0000 & 27.0094 \\
\hline$M R$ * & 0.0 & 0.0 & $10.3 \%$ & $18.10 \%$ & $14.0 \%$ & ND & ND \\
\hline \multicolumn{8}{|l|}{1 year to 6 years } \\
\hline BCG & $99.7 \%$ & $99.6 \%$ & $99.6 \%$ & $97.0 \%$ & $98.6 \%$ & 0.0236 & 11.2761 \\
\hline Polio & $89.4 \%$ & $88.2 \%$ & $97.2 \%$ & $83.0 \%$ & $75.0 \%$ & 0.0000 & 47.8885 \\
\hline Pentavalent & $80.4 \%$ & $81.4 \%$ & $90.5 \%$ & $46.81 \%$ & $67.1 \%$ & 0.0000 & 32.2985 \\
\hline Rotavirus & $90.3 \%$ & $89.6 \%$ & $85.7 \%$ & $67.9 \%$ & $35.0 \%$ & 0.0000 & 198.8064 \\
\hline Pneumococcus & $17.1 \%$ & $14.4 \%$ & $8.3 \%$ & 9.8 & $4.6 \%$ & 0.0009 & 18.6351 \\
\hline Influenza & $29.1 \%$ & $20.0 \%$ & $16.4 \%$ & $5.4 \%$ & $7.1 \%$ & 0.0000 & 55.0644 \\
\hline MMR & $74.0 \%$ & $75.2 \%$ & $76.3 \%$ & $61.0 \%$ & $67.7 \%$ & 0.0035 & 15.6807 \\
\hline \multicolumn{8}{|l|}{ Over 6 years } \\
\hline BCG & $100.0 \%$ & $100.0 \%$ & $100.0 \%$ & $100.0 \%$ & $98.8 \%$ & 0.1006 & 7.764 \\
\hline Polio & $92.9 \%$ & $98.1 \%$ & $97.7 \%$ & $98.2 \%$ & $82.5 \%$ & 0.0000 & 35.4147 \\
\hline Pentavalent & $84.4 \%$ & $91.6 \%$ & $90.5 \%$ & $91.67 \%$ & $82.1 \%$ & 0.0551 & 9.2551 \\
\hline Rotavirus & $91.8 \%$ & $94.8 \%$ & $94.2 \%$ & $97.3 \%$ & $68.0 \%$ & 0.0000 & 60.8297 \\
\hline Pneumococcus & $24.7 \%$ & $30.0 \%$ & $24.0 \%$ & $20.8 \%$ & $9.1 \%$ & 0.0108 & 13.0939 \\
\hline Influenza & $28.7 \%$ & $33.1 \%$ & $21.8 \%$ & $6.7 \%$ & $11.8 \%$ & 0.0000 & 33.331 \\
\hline MMR & $82.1 \%$ & $93.6 \%$ & $90.6 \%$ & $85.1 \%$ & $75.6 \%$ & 0.0005 & 19.8696 \\
\hline
\end{tabular}

Immunization decline above 30\% was observed for other vaccines. The only exception was for the MR vaccine, which wasn't administrated the years 2015 and 2016 for children under 12 months old because there was no measles epidemic in those years. Importantly, in 2019 no children less than 1 year was vaccinated against influenza, and only $3.5 \%$ received the pneumococcal vaccine (Table 5).

A significant coverage decline is observed for the group 1 to 6 years old, with the largest decline between 2015 and 2019 in the cases of rotavirus $(55.4 \%)$ and influenza $(22 \%)(p=0.0000)$. Significantly, the MMR (measles, rubella, mumps) coverage dropped by $6.3 \%$ between 2015 and 2019 ( $p=0.0035)$. (Table 5).

For the group older than 6 years, significant drop in vaccination coverage was only observed for the case of rotavirus $23.8 \%$ and influenza $16.8 \%(p=0.0000)$. (Table 5)

\section{Discussion}

Immunization is an essential component of the human right to health, and is the responsibility of individuals, communities and governments. It is estimated that vaccination prevents 2.5 million deaths each year. Children immunized and protected from the threat of diseases preventable 
by vaccination have the opportunity to develop and more likely to reach their full potential. These advantages are further reinforced by vaccination of adolescents and adults. As part of an intervention plan to prevent and control diseases, vaccines and immunization are an essential investment in the future of a country and even of the world (13).

Vaccination is one of the most beneficial strategies in public health. Scientific, technological and social advances offer great opportunities to expand basic vaccination schedules. (3).

Maximizing the impact of vaccination on public health requires a number of conditions. Among these factors are the need to generate more preventive awareness among the population, the imperative to ensure political support for the healthcare sector in terms of allocation of financial resources for the introduction of new vaccines, and the need to counteract the effect of the anti-vaccine groups (3).

By 2010 WHO established the general goals of the decade 2011-2020: 1. To get a polio-free world 2.- To meet vaccination coverage targets in all countries, regions and communities, 3.- To develop and introduce new and improved vaccines and technologies 4.- To overcome the number 4 Millennium Development Goal on reducing child mortality. If these specific immunization goals were achieved, hundreds of millions of cases and millions of future deaths would be avoided by the end of 2020, and thousands of millions of dollars of productivity would be earn, and immunization would help to achieve the target of the 4th Millennium Development Goal by reducing child mortality (13).

In May 2017, Health Ministers of 194 countries adopted a new resolution to strengthen vaccination in order to achieve the objectives of the Global Vaccine Action Plan. In this resolution, countries are encouraged to show leadership and more robust governance over national immunization programs, to strengthen monitoring and surveillance in order to ensure the use of updated data to guide strategic decisions, to optimize program's performance and impact of immunization. In addition, it is recommended to expand immunization services beyond infancy, to mobilize internal funds and to strengthen international cooperation to achieve the objectives of the Global Vaccine Action Plan (3).

It is estimated that in 2016, 19.5 million infants worldwide were outside the scope of routine immunization services, like the third dose of the triple bacterian (DTP3). About $60 \%$ of them live in 10 countries: Angola, Brazil, Ethiopia, India, Indonesia, Iraq, Nigeria, Pakistan, Democratic Republic of Congo and South Africa (3).

Monitoring data at the national level is critical to assist countries prioritizing and tailoring vaccination strategies and operational plans to fill immunization gaps, and to get people all the shots which can save their lives (3).

According to the latest Pan American Health Organization (PAHO) report on immunization coverage in Venezuela, 2017 vaccination coverage was $100 \%$ for BCG, $66 \%$ for DTP (3rd dose), $79 \%$ for polio (3rd dose), $95 \%$ for MMR, and $18 \%$ for rotavirus $(14,15)$.

In the present study it was observed a low coverage for all vaccines in 2019 - a situation already evidenced by WHO in 2017-. An average decrease in vaccine coverage of $21.5 \%$ from 2015 to 2019 was verified. The smallest decline in coverage was for the BCG vaccine which only decreased by $6.3 \%$. On the contrary, a significant decline in coverage in the years evaluated was found for the other vaccines, being the more important coverage decline for the rotavirus vaccine (52.2\%) followed by influenza, where the drop was $21.1 \%$. These deficits are exacerbated when they are analyzed by age group. The worst case is for children under one year old: coverage for rotavirus dropped $69.2 \%$, and influenza vaccine reaches $0 \%$ coverage in the studied population. The coverage drop was less marked for children aged 1 to 6 years, although there was also a significant drop in rotavirus and influenza vaccination rate. In children older than 6 years, significant differences were found in terms of vaccination coverage, except for the case of BCG and Pentavalent vaccine.

Even though pneumococcal vaccine was included in 2013 in the EPI in Venezuela, and began to be implemented in May 2014, a very low coverage of this vaccine is observed. Only $16 \%$ of patients had access to full immunization schedule, and a coverage drop of $16.1 \%$ is found between 2015 and 2019. This drop is more accentuated for the group of children under 1 year old: in this age group coverage was only $3.5 \%$ in 2019.

The scenario just described contrasts with studies in other countries in the region, where it is evidenced lower coverage for children older than 6 years old. For instance, in 2016 Carme Saperas Pérez (7) evaluated vaccination coverage by age group in a municipality of Apartado, Colombia, evidencing correct coverage of hepatitis B and BCG in newborns, with figures ca. 100\%. However, as age increases, declining immunization coverage is observed, being for pentavalent in children older than 1 year of $90.7 \%, 90.15 \%$ polio, MMR $88.86 \%$, yellow fever figures critical booster vaccination coverage of DTP and MMR at 5 years of age, which differs from our study, where better vaccination coverage was observed in children aged 12 months to 6 years and in critical figures in children under 12 months old. (7)

In its report of 2017, the Roundtable for Poverty Reduction (Peru) assessed vaccination coverage for full compliance with the basic vaccination schedule in children under 3 years. They found an increase in the overall vaccination coverage from $55.7 \%$ in 2014 , to $69.4 \%$ in 2015 (up 13 percentage points increment) for children under 1 year old -where timely vaccination is a reflection of the quality of care-. The increase in coverage continues in 2016 , reaching levels of $74.0 \%$ (up 5 percentage points). In the first half of $201775.5 \%$ coverage (16) was reached.

Page $7 / 10$ 
Decline in vaccination coverage is influenced by several factors, one of the most important being vaccine availability. According to Venezuela's 2017 EPI report, produced and published by PAHO / WHO, vaccine shortages in Venezuela have increased from 2012 to 2017, with shortages reported in 2012 for hepatitis B and polio vaccines, while in 2017 shortages of pneumococcal conjugate, DTP, yellow fever, hepatitis B, Hib, Influenza, IPV, oral polio and rotavirus vaccines are reported (15). This would explain the decrease in compliance with the recommended immunization schemes observed in the present work, when comparing data of 2015 and 2019.

Low vaccination coverage have caused the rise in previously eradicated diseases as measles and diphtheria. In Venezuela, between epidemiological week 26th of 2017 and the 52th of 2018, 9,116 suspected cases of measles were reported (1,307 in 2017 and 7809 in 2018$)$ of which 6,202 were confirmed (727 in 2017 and 5,475 in 2018). The cumulative incidence rate in the country from 2017 to 2019 is 19.6 cases per 100,000 inhabitants (17); despite this epidemic, it was observed a decline in coverage against measles in the years studied, dropping to $61 \%$ coverage for 2019 .

There is also an epidemic of diphtheria in Venezuela that started in July 2016 and which is still active in March $2019.2,512$ suspected cases of diphtheria have been registered up to the 2 nd epidemiological week of the year 2019, of which 1,559 were confirmed, with 270 deaths (18). However, a decrease in diphtheria's vaccine coverage was evidenced in the present study. We found a decrease in pentavalent coverage of $80.2 \%$ for 2015 and $59.9 \%$ for 2019 , this decrease being more pronounced in children under 1 year old, for whom coverage for 2019 was $30.6 \%$.

Immunizations represent, after clean, safe water, the most effective way of preventing infectious diseases, one of the most outstanding public health achievements in child health. Hence, the importance of knowing their application cannot be overestimated; systematic vaccinations, particularly at ages in greater risk, will dramatically decrease the incidence of infectious diseases.

The main limitation of the study was to obtain from parents the information on the immunization schedule that many of them did not know the information and lost the vaccination record of their children.

\section{Conclusions}

Even though vaccination has been one of the most important measures of intervention in reducing child mortality over the past 40 years, our findings determine the absence of optimum vaccination coverage in patients enrolled in the present study.

It is necessary to strengthen preventive medicine; the EPI has to be well implemented to be able to prevent outbreaks of vaccine-preventable diseases, which contrasts with Venezuela's current situation.

Low vaccination rates suggest that the goal of eliminating measles and diphtheria is likely to remain elusive, unless greater efforts to improve vaccination coverage are made

As vaccination is less expensive than the burden of diseases, it is essential (and cost-beneficial) to strengthen the national immunization program.

\section{Abbreviations}

PAHO

Pan American Health Organization

HUC

Hospital Universitario de Caracas

WHO

World Heald Organization

EPI

Expanded Program of Inmmunization

MPPS

Ministry of Popular Power for Health (Ministerio del Poder Popular para la Salud)

\section{Declarations}

Ethics approval: The study was approved by the Ethics Committee of the Hospital Universitario de Caracas on February 2, 2015, CBE N ${ }^{\circ}$ 03/2015 signed for doctor Claudio Urosa Bioethics Committee Coordinator.

Consent to participate: informed consent was given in writing by the parent or guardian for all children participating in the study 
Consent for publication: Not applicable

Availability of data and materials: the datasets used and/or analyzed during the current study are available from the corresponding author on reasonable request.

Competing interests: The authors declare that they have no competing interests

Funding: financing with own funds

\section{Authors' contributions}

TD: study direction, perform study design, analyzed the patient data and write the manuscript

IM: Perform study design and analyzed the patient data and write the manuscript

AS, MJR, JG, RT and MS: collected, analyzed and interpreted the patient data

BR and AT: analyzed data, write and revised the manuscript.

All authors read and approved the final manuscript.

Acknowledgements: To all residents of the HUC Department of Pediatrics who assisted in data collection

\section{References}

1. Black R, Laxminarayan R, Temmerman M, Walker N. Reproductive, Maternal, Newborn, and Child Health. Volume 2. Third Edition. Washington The World Bank, 2016. p. 187.

2. De Oliveira M, Zangiaconi E, Yazlle J. Factors associated With children vaccination coverage in children under 5 years in Angola. Rev Saúde Public. 2014;48(6):906-15.

3. Vaccination coverage [Internet] World Health Organization. 2018. [Cited 22 March 2018] Available in http://www.who.int/mediacentre/factsheets/fs378/es/.

4. Wanjala S. Determinants of Immunization coverage in Kenya. [Final dissertation on the Internet]. [Nairobi]: University of Nairobi; 2014. [Cited November 2014]. Available in http://erepository.uonbi.ac.ke/.

5. Shemwell S, Peratikos M, Gonzalez L, Renom- Llonds M, Boon A, Martinho S, et al. Determinants of full vaccination status in children aged 12-23 months in Gurué and Milanpe districts, Mozambique: results of a population -based cross- sectional survey. Int Health. 2017;9:234-42.

6. Dunn A, Black C, Arnold J, Brodine S, Waalen J, Binkin N. Childhood Vaccination Coverage Rates Arnong Military Dependents in the United States. PEDIATRICS. 2015;135(5):e1156 e1148-

7. Saperas C. Diagnostic Technical Report on the status of immunization coverage in children under 5 years. [Internet]. Apartado Antioquia Department, Colombia. [Cited February 2016]. Available in: https://ddd.uab.cat/pub/trerecpro/2016/149148/.

8. Figueiredo A, Jhonston L, Smith D, Agarwal S, Larson M, Jones N. Forescasted trends in vaccination coverage and socioeconomic factors correlations With global time - series analysis over 30 years. Glob Health Lancet. 2016;4:e726-35.

9. Tregnaghi M, Sosa A, editors. Manual vaccines in Latin America [monograph on the Internet]. Latin American Society of Pediatric Infectious Diseases SLIPE; 2014 [Cited 24 February 2019] Available: https://www.vaxeen.com/files/Manual\%20de\%20Vacunas\%20\%20Edici\%C3\%B3n\%202014\%20-\%20SLIPE.pdf.

10. Ministry of Popular Power for Health. Report and Accounts 2015 Caracas [Internet]. Popular Ministry for Health, 2015: 112. [Cited 24 February 2019] Available: https://es.scribd.com/doc/302207521/Memoria-del-Ministerio-de-Salud-2015.

11. Venezuelan Observatory of Health. The complex emergency suffered by Venezuela and the performance of the Pan American Health Organization [Internet] Center for Development Studies Central University of Venezuela [Cited February 24, 2019] Available: https://www.ovsalud.org/noticias/2018/emergencia-venezuela-organizacion-panamericana-salud/.

12. Carrizo J, Drummond T, Betancourt A, Izaguirre J, Perez, Figueroa D, Rísquez A, Levy J. E. Sileo immunization schedule for children and adolescents in July 2018. Socidad Venezolana de Puericultura y Pediatría. [Internet] Venezuelan Society Childcare and Pediatrics [Cited September 18, 2018] Available: http://www.svpediatria.org/secciones/publicaciones/esquema-de-inmunizacion/.

13. WHO Global Action Plan on vaccines [Internet] World Health Organization. 2013 [Cited February 28, 2019] Available: https://apps.who.int/iris/bitstream/handle/10665/85398/9789243504988_spa.pdf;jsessionid=C73986AA1585B930F91C9E9F2591F09F? sequence $=1$. 
14. WHO Vaccine Coverage [Internet] World Health Organization [Cited February 28. 2019] Available: https://www.who.int/es/news-room/factsheets/detail/immunization-coverage.

15. $\mathrm{PAHO} / \mathrm{WHO}$

PAHO / WHO. Country Report EPI, Venezuela, 2017 [Internet] Pan American Health Organization 2018. [Cited February 28, 2019] Available: https://www.paho.org/hq/index.php?option=com_docman\&view=download\&category_slug=perfiles-paises-im-1809\&alias=4803venezuela-perfil-pais-803\&ltemid=270\&lang=es.

16. Roundtable for Poverty Reduction. Status of vaccine coverage for children under 3 years.-the first half 2017 [Internet] Monitoring Team concerted Health Policy. Sub Group "Immunization in children under 5 years" Report No. 2-2017-SC / MCLCP [Cited 28de February 2019] Available:

https://www.mesadeconcertacion.org.pe/sites/default/files/archivos/2017/documentos/09/reportevacunasgtsalud240817vrev6.pdf.

17. Pan American Health Organization / World Health Organization. Epidemiological update: measles. March 4, 2019 [Internet] Washington, DC: Pan American Health Organization 2019. [Cited 13 March 2019] Available: https://www.paho.org/hq/index.php? option=com_docman\&view=download\&category_slug=2019-3\&alias=47909-4-de-marzo-de-2019-sarampion-actualizacionepidemiologica\&ltemid=270\&lang=es.

18. Pan American Health Organization / World Health Organization. Epidemiological update: Diphtheria. January 22,2019 [Internet] Washington, DC: Pan American Health Organization 2019. [Cited 13 March 2019] Available: https://www.paho.org/hq/index.php? option=com_docman\&view=download\&category_slug=2019-3\&alias=47971-8-de-marzo-de-2019-difteria-actualizacionepidemiologica\&ltemid=270\&lang=es.

\section{Supplementary Files}

This is a list of supplementary files associated with this preprint. Click to download.

- STROBEchecklistStatusofvaccinecoverage.doc 